Journal of

Cognitive Neuroscience

\title{
Auditory attention causes gain enhancement and frequency sharpening at successive stages of cortical processing - evidence from human EEG
}

\begin{tabular}{|r|l|}
\hline Journal: & Journal of Cognitive Neuroscience \\
\hline Manuscript ID & JOCN-2017-0047.R2 \\
\hline Manuscript Type: & Original \\
\hline Date Submitted by the Author: & 15 -Jan-2018 \\
\hline Complete List of Authors: & $\begin{array}{l}\text { de Boer, Jessica; MRC Institute of Hearing Research, School of Medicine, } \\
\text { University of Nottingham, } \\
\text { Krumbholz, Katrin; MRC Institute of Hearing Research, School of Medicine, } \\
\text { University of Nottingham, }\end{array}$ \\
\hline Keywords: & $\begin{array}{l}\text { Human auditory cortex, attentional modulation, repetition suppression, } \\
\text { stimulus-specific adaptation, cortical auditory-evoked potentials (CAEPs) }\end{array}$ \\
\hline
\end{tabular}


1 Title: Auditory attention causes gain enhancement and frequency sharpening at successive

6 Authors: Jessica de Boer, Katrin Krumbholz

7 Affiliation: MRC Institute of Hearing Research at the School of Medicine, University of 8 Nottingham.

Corresponding author: Jessica de Boer, MRC Institute of Hearing Research at the School of Medicine,

University of Nottingham, Science Road, University Park, Nottingham, NG7 2RD.

E-mail:Jessica.deBoer@nottingham.ac.uk

Keywords: Human auditory cortex, attentional modulation, repetition suppression, stimulusspecific adaptation, cortical auditory-evoked potentials (CAEPs).

ABSTRACT

Previous findings have suggested that auditory attention causes not only enhancement in neural processing gain, but also sharpening in neural frequency tuning in human auditory cortex. The current study was aimed to reexamine these findings, and investigate whether attentional gain enhancement and frequency sharpening emerge at the same or different processing levels, and whether they represent independent or cooperative effects. For that, we examined the pattern of attentional modulation effects on early, sensory-driven cortical auditory-evoked potentials (CAEPs) occurring at different latencies. Attention was manipulated using a dichotic listening task and was thus not selectively directed to specific frequency values. Possible attention-related changes in frequency tuning selectivity were measured with an EEG adaptation paradigm. Our results show marked disparities in attention effects between the earlier N1 CAEP deflection and the subsequent P2 deflection, with the N1 showing a strong gain enhancement effect, but no sharpening, and the P2 showing clear evidence of sharpening, but no independent gain effect. They suggest that 
33 attentional modulation mechanism, which appears to increase the representational bandwidth of attended versus unattended sounds.

INTRODUCTION

There is manifold evidence that attention causes top-down modulation of sensory-driven, or "exogenous", cortical responses (e.g., Fritz, Shamma, Elhilali, \& Klein, 2003; Hillyard, Hink, Schwent, \& Picton, 1973; Spitzer, Desimone, \& Moran, 1988; reviewed in: Fritz, Elhilali, David, \& Shamma, 2007; Mangun \& Hillyard, 1995), but the mechanisms underlying this modulation still remain unclear. Two alternative models have been proposed: the "gain enhancement" model assumes that attention increases neuronal responsiveness to the attended stimulus (Hillyard, Vogel, \& Luck, 1998; McAdams \& Maunsell, 1999), and the "sharpening" model, that attention increases neuronal tuning selectivity (Atiani, Elhilali, David, Fritz, \& Shamma, 2009; Fritz et al., 2003; Spitzer et al., 1988). The current study aimed to test whether or how these models apply to the auditory domain. In particular, we wanted to test (i) whether exogenous auditory responses are really affected by attentional sharpening, and, if so, (ii) how gain enhancement and sharpening relate within the context of the auditory processing hierarchy: do they occur at the same or different processing levels, and do they operate cooperatively or independently of one another?

Numerous earlier studies have found non-invasively recorded auditory cortical responses to be larger when the evoking sound is attended, rather than unattended (EEG/MEG: Fujiwara, Nagamine, Imai, Tanaka, \& Shibasaki, 1998; Hillyard et al., 1973; Hillyard et al., 1998; Woldorff et al., 1993; Woldorff \& Hillyard, 1991; fMRI: Jäncke, Mirzazade, \& Shah, 1999), and have generally interpreted this finding within the context of a gain enhancement mechanism. More recently, however, it has been suggested that auditory attentional modulation also involves sharpening (Ahveninen et al., 2011; Kauramaki, Jääskeläinen, \& Sams, 2007; Okamoto, Stracke, Wolters, Schmael, \& Pantev, 2007). To demonstrate sharpening, the previous studies have used paradigms involving "notched noise" (NN) masking, a technique that has been used extensively in behavioral measurements of auditory frequency selectivity (e.g., Glasberg \& Moore, 1990). NN masking requires the subject to attend to a fixed-frequency tone, whilst trying to ignore a concurrently presented broadband noise with a spectral notch centered on the tone frequency. When the notch is narrow enough so that the tone response is partially 
obscured, or "masked", by the noise response, the size of the unobscured portion of the tone response (over and above the noise response) should depend on the tuning selectivity of the tone-responsive neurons (Sams \& Salmelin, 1994), and should thus be sensitive to any sharpening in tuning selectivity induced by attention. Consistent with this expectation, the previous studies have found greater attentional enhancement of the tone response size when the notch was narrower than when it was wider (Kauramaki et al., 2007; Okamoto et al., 2007), or when the masking noise was omitted altogether (Ahveninen et al., 2011). Arguably, however, this finding could also be explained in terms of gain enhancement. This is, because the tone was presented at a fixed intensity and would thus have been less audible when presented in a narrow-notched noise. As a result, the unattended tone response size would have been smaller, and the attentional task would have been more difficult to perform. Earlier findings (Alho, Woods, Algazi, \& Näätänen, 1992; Boudreau, Williford, \& Maunsell, 2006; Schwent, Hillyard, \& Galambos, 1976a, 1976b) suggest that both factors should have led to greater attentional gain enhancement, thus mimicking the effect of attentional sharpening.

To avoid these confounds, the current study manipulated attention and measured tuning selectivity independently using dichotic listening and adaptation, respectively. Tone or noise sequences were presented concurrently to opposite ears ("Ipsi" and "Contra" in Fig. 1A) and subjects were asked to alternately attend to one or other sequence. Cortical auditory-evoked potentials (CAEPs) were recorded in response to the tone sequences, and the tone frequency was varied randomly from trial to trial to vary the degree of adaptation between successive tones. Adaptation refers to the suppression in neuronal response when the same or similar stimulus is presented repeatedly (hence also referred to as "repetition suppression"; Grill-Spector, Henson, \& Martin, 2006). Adaptation is ubiquitous across many sensory domains and has become a popular tool for probing functional properties of neuronal populations, particularly in the visual domain (reviewed in Snow, Coen-Cagli, \& Schwartz, 2017; Webster, 2015), but to a lesser degree also in the auditory domain (e.g., Briley, Breakey, \& Krumbholz, 2013; Edmonds \& Krumbholz, 2014; Hewson-Stoate, Schonwiesner, \& Krumbholz, 2006; Magezi \& Krumbholz, 2010; Salminen, May, Alku, \& Tiitinen, 2009). Under the assumption that adaptation is caused by neuronal fatigue (mediated by synaptic depression or somatic after-hyperpolarization; Briley \& Krumbholz, 2013; Lanting, Briley, Sumner, \& Krumbholz, 2013), the degree of adaptation between two 
successive tones should depend on the degree of overlap between the neuron populations responsive to the tones, and this, in turn, should depend on the neurons' frequency tuning selectivity. Figure $1 \mathrm{~B}$ shows predictions of how the adapted tone response sizes might be affected by attentional gain enhancement and sharpening effects. The predictions are based on a simple neuron population model, with model neurons tuned for frequency and subject to activity-dependent adaptation, or fatigue (see Methods for model details). Due to adaptation, the aggregate population response size to the current tone is predicted to increase with increasing frequency separation of the preceding tone, regardless of attention condition (right panels in Fig. 1B). Under the assumption of a pure gain enhancement mechanism (with multiplicative gain; top row in Fig. 1B), attention is predicted to increase the population response size equally across all frequency separations (if response size is expressed in logarithmic units), leaving the shape of the response size function unchanged. In contrast, a pure sharpening mechanism (middle row in Fig. 1B) is predicted to increase the initial slope of the response size function (at small frequency separations), but also, to cause an overall suppression in response size across all frequency separations. The suppression arises, because, as the neurons' tuning selectivity increases, fewer neurons are activated and thus the aggregate population response size decreases. In order to avoid suppression, the sharpening has to be combined with a gain enhancement such that the aggregate response size remains constant (Fig. 1B, bottom row). As a result, the initial slope of the response size function is again predicted to steepen, but the response size now remains unchanged at zero and large frequency separations (when the responses to the successive tones overlap either completely or not at all; see left and middle panels in Fig. 1B).

\section{$* * *$ insert Fig 1 about here ${ }^{* * *}$}

The previous studies that have used NN masking to investigate auditory attentional modulation mechanisms (Ahveninen et al., 2011; Kauramaki et al., 2007; Okamoto et al., 2007) have focused exclusively on the prominent N1 deflection of the CAEPs (Näätänen \& Picton, 1987). Here, we also examined the preceding and following P1 and P2 deflections, which, like the N1, are exogenous, and thus presumably represent earlier and later stages of sensory-driven auditory processing. Our results suggest that gain enhancement and sharpening represent cooperative components of a hierarchically distributed auditory attentional modulation mechanism, affecting different sensory-driven processing levels: the 
earliest observed attention effects (in the N1) appeared to be pure gain enhancement effects, whilst sharpening effects appeared to emerge only at later processing levels (in the P2). Our results suggest that gain enhancement and sharpening might work together to increase the representational bandwidth, or "data rate", of attended over unattended auditory information.

\section{MATERIALS AND METHODS}

\section{Participants}

23 subjects ( 7 male; mean age $=23.1, \mathrm{SD}=3.8$ years) participated after having given written informed consent. All subjects had hearing thresholds at or below $20 \mathrm{~dB} \mathrm{HL}$ at all audiometric frequencies $(250-8000 \mathrm{~Hz})$, and had no history of audiological or neurological disease. The experimental procedures accorded with the Declaration of Helsinki (Version 6, 2008) and were approved by the Ethics Committee of the University of Nottingham School of Psychology, but were not formally pre-registered online in accordance with the 2014 amendment to the declaration.

\section{Stimuli and procedure}

During the EEG experiment, subjects were comfortably seated in an electrically shielded, sound-attenuating booth (IAC Acoustics, Winchester, United Kingdom). The experiment consisted of four runs with short breaks in between. In three runs, referred to as "active runs", subjects were required to alternately attend to tone or noise sequences, presented to opposite ears, and detect infrequent targets within the attended ear. The to-be-attended ear was indicated by visual instruction and was switched every $\sim 2$ min. The ear of presentation of the tone and noise sequences was counterbalanced across subjects. The active runs lasted about 12 min each. In the remaining run, referred to as "passive run", the stimuli were presented passively whilst the subjects watched a silent sub-titled movie of their own choice to remain alert. The duration of the passive run was matched to the total duration for which subjects attended to each ear over the three active runs (i.e., $3 \times 6 \mathrm{~min}=$ $18 \mathrm{~min})$. The active and passive runs were played consecutively, in counterbalanced order across subjects.

The tones ("Ipsi" in Fig. 1A) had a duration of $100 \mathrm{~ms}$, including 20-ms cosinesquared onset and offset ramps, and were presented at a fixed stimulus onset interval (SOI) of $500 \mathrm{~ms}$. A fixed SOl was used, because varying it would have varied the degree of 
161 adaptation between successive tones (Lanting et al., 2013) and thus confounded the tuning

162

163

164

165

166

167 selectivity measurement. The tone frequencies were distributed equally between four different values, which were by 0, 75, 150 and 300 cents higher than $1000 \mathrm{~Hz}(1000,1044$, 1091, $1189 \mathrm{~Hz}$ ). The tone sequences were pseudo-random de Bruijn sequences consisting of 256 items each (lasting $2 \mathrm{~min}$ ). They were designed such that not only each frequency individually, but also each possible combination of two, three or four consecutive frequencies occurred an exactly equal number of times $(64,16,4$ and 1, respectively; Brimijoin \& O'Neill, 2010).

The noise stimuli ("Contra" in Fig. 1A) were generated from equally exciting noise (with equal energy falling in each auditory filter; Glasberg \& Moore, 2000), which was boxcar filtered between 2000 and $3000 \mathrm{~Hz}$. They had a duration of $200 \mathrm{~ms}$, and were amplitudemodulated with a waxing amplitude envelope consisting of linear onset and offset ramps lasting 150 and $50 \mathrm{~ms}$, respectively. The SOI of the noises was randomized between 666 and $966 \mathrm{~ms}$ (mean: $816 \mathrm{~ms}$ ) to decorrelate the onset times of the tones and noises across the two ears.

The tone targets were distinguished from the non-target tones by a linearly rising frequency ramp (the non-target tones had a steady frequency; right panel in Fig. 1A). They were presented randomly with a probability of $7.5 \%$, with the constraint that every two successive target tones were separated by at least four non-target tones. The noise targets were time-reversed versions of the non-target noises (non-targets were waxing, and targets were waning noises; Fig. 1A; idea taken from Cusack, Deeks, Aikman, \& Carlyon, 2004). They were presented with a probability of $10 \%$ and separated by at least two non-target noises. On average, both the tone and noise targets occurred about 20 times within each $\sim 2$-min period (targets were presented within both the attended and unattended sequences).

All stimuli were generated digitally using Matlab (The Mathworks, Natick, MA, USA) and digital-to-analogue converted with a 24.414-kHz sampling rate and 24-bit amplitude resolution using TDT System 3 (Tucker Davis Technologies, Alachua, FL, USA) consisting of an RP2.1 real-time processor and an HB7 headphone buffer. Both the tone and noise stimuli were presented at a sound pressure level (SPL) of $65 \mathrm{~dB}$ using Sennheiser HD-280 Pro circumaural headphones (Sennheiser, Wedemark, Germany).

\section{EEG recordings}


CAEPs were recorded with $33 \mathrm{Ag} / \mathrm{AgCl}$ ring electrodes (EASYCAP, Herrsching, Germany), placed according to the standard 10-20 layout, and a BrainAmp DC EEG amplifier (Brain Products, Gilching, Germany). Skin-to-electrode impedances were maintained below $5 \mathrm{k} \Omega$ throughout the recordings. The recording reference was the vertex $(\mathrm{Cz})$ channel and the ground was placed on the central forehead (AFz). The electrode signals were sampled at 500 $\mathrm{Hz}$ and bandpass-filtered online between 0.1 and $250 \mathrm{~Hz}$ using BrainVision Recorder (Brain Products). Only the responses to the non-target tones were analyzed further.

\section{EEG data analysis}

The EEG data were first pre-processed using the EEGLAB toolbox (Delorme \& Makeig, 2004), which runs under Matlab. First, they were lowpass filtered at $35 \mathrm{~Hz}$ using a -48-dB/oct zerophase IIR filter, and then they were re-referenced to average reference and segmented into 500-ms epochs ranging from $100 \mathrm{~ms}$ before, to $400 \mathrm{~ms}$ after the onsets of the non-target tones. Epochs containing unusually large amplitudes across electrodes (joint probability larger or equal to three standard deviations) were rejected automatically. The remaining epochs were submitted to an independent component analysis (extended infomax algorithm). Components representing eye blinks, lateral eye movements and electro-cardiac activity were removed by manual inspection of the components' temporal traces and scalp topographies.

Activity during the baseline period of the tone responses (before the tone onset) was both highly non-stationary and also considerably larger for attended than unattended trials (Fig. 2A), suggesting the presence of longer-lasting endogenous activity from preceding trials (Woldorff, 1993). To minimize the effect of this activity on the analysis of the discernible exogenous deflections (P1, N1 and P2; Fig. 2A), we baseline-corrected each deflection separately, using a different baseline window (referred to as "deflection-specific" baseline correction). All windows were given a minimal duration of only $8 \mathrm{~ms}$. The windows for the $\mathrm{N} 1$ and P2 were centered at the peaks of the respective preceding, opposite-polarity deflections (P1 and N1, respectively), thus effectively creating a peak-to-peak difference. This would be expected to minimize any unipolar activity associated with endogenous attentional processing, such as the so-called "processing negativity" (Näätänen, 1990), which would affect opposite-polarity deflections in opposite directions, and thus cancel in the peak-to-peak difference. The window for the P1 was located at the tone onset (around 0 $\mathrm{ms})$, close to the P1 deflection start. The baseline correction was performed separately for 
224

225

226

227

228

229

230

231

232

233

234

235

236

237

238

239

240

241

242

243

244

245

246

247

248

249

250

251

252

253

254

255

each subject and analyzed condition. The baseline-corrected deflections will be referred to as $\mathrm{P} 1_{0}, \mathrm{~N} 1_{\mathrm{P} 1}$ and $\mathrm{P} 2_{\mathrm{N} 1}$ to indicate the differences in baseline window (see Fig. $2 \mathrm{~B}$ ).

The $\mathrm{P} 1_{0}, N 1_{\mathrm{P} 1}$ and $\mathrm{P} 2_{\mathrm{N} 1}$ peak amplitudes were measured both from the original sensor data, and also from source waveforms derived from source models fitted to each deflection peak. The sensor data were evaluated at the sensors that showed the largest unattended deflection peaks on average ( $\mathrm{Fz}$ for the $\mathrm{P} 1_{0}$ and $\mathrm{N} 1_{\mathrm{P} 1}$, and $\mathrm{Cz}$ for the $\left.\mathrm{P} 2_{\mathrm{N} 1}\right)$ and referenced to the linked mastoids (average of TP9 and TP10). The source models were fitted to the unattended conditions only (when subjects attended to the noise sequences in the opposite ear or watched a silent movie) to create a spatial filter for exogenous auditory cortical activity. They were implemented in the Brain Electrical Source Analysis software, version 5.3 (BESA, Gräfelfing, Germany), and each consisted of two hemispherically symmetric regional equivalent current dipoles (ECDs; Scherg \& Ebersole, 1993), with a fourshell ellipsoidal volume conductor as head model. First, the ECD locations were fitted to a 30-ms window centered at the relevant deflection peak in the grand-average response across all subjects and unattended conditions. Then, the ECDs were then re-oriented individually for each subject to maximize the peak source strength along their first dipole direction, and the resulting reoriented first dipole directions were used to extract source waveforms for each individual and condition. The source waveforms showed no significant hemispheric differences, and were thus averaged across hemispheres.

The $P 1_{0}, N 1_{P 1}$ and $P 2_{N 1}$ peak amplitudes were either averaged across all tone frequencies, or evaluated separately for each absolute frequency separation, $\Delta \mathrm{F}$, between the current and preceding tones, which could take one of four values $(0,75,150$ or 300 cents). On average, the number of trials available for each absolute frequency separation and each subject was 391 (range: 347-414), 479 (409-517), 481 (428-507) and 241 (208-258) when subjects attended to the tone sequences, 397 (374-419), 490 (463-512), 491 (465-508) and 245 (231-260) when they attended to the noise sequences, and 380 (330-406), 469 (421-499), 467 (412-506) and 236 (212-248) when they watched a silent movie (passive run).

\section{Statistical analyses}

Statistical analyses were conducted using $R$ (R Core Team, 2013). Both the behavioral (hit/false-alarm rates and reaction times for target detection) and CAEP data (deflection peak amplitudes) were evaluated with linear mixed-effects models (nlme package; Pinheiro, 
Bates, DebRoy, Sarkar, \& Team, 2017). The CAEP peak amplitudes were first converted to logarithmic units.

Homogeneity of variance was tested using Levene's test (car package; Fox \& Weisberg, 2011), and normality using quantile-quantile plots of the model residuals. Where variance homogeneity was violated (i.e., the residuals were significantly different across factor levels), each observation was weighted by the inverse of the variance for the respective factor level. This reduces the influence of noisier data points on the model fit. Normality was achieved by log-transformation (applied to the false-alarm rates and reaction times). Any overly influential data points were identified using Cook's distance and excluded.

In the models of the CAEP peak amplitudes, the linear frequency separation covariate $(\Delta \mathrm{F})$ was shifted downwards by 150 cents $(\Delta \mathrm{F} \rightarrow \Delta \mathrm{F}-150$ cents) to reduce collinearity with the quadratic covariate $\left(\Delta \mathrm{F}^{2}\right)$. Next to the fixed effects, all models also contained by-subjects random intercepts and fixed-factor slopes. The fixed effects were fitted using maximum likelihood (ML) estimation, and the random effects using restricted ML (REML) estimation. Random effects were tested using log-likelihood ratio tests. Random effects that failed to produce a significant improvement in model fit were omitted. Fixed effects were evaluated using conditional F-tests following the strategy described in Pinheiro and Bates (2000). Despite some missing data points, the number of data points were sufficiently similar across the various combinations of factor levels to allow type-III (marginal) tests to be evaluated for all included fixed effects. Significant fixed effects were post-hoc tested using Tukey's honestly significant difference (multcomp package; Hothorn, Bretz, \& Westfall, 2008).

\section{Neuron population model of attentional modulation effects}

Predicted effects of gain enhancement and sharpening were derived with a neuron population model, where each neuron was tuned to a different characteristic frequency, $f_{c}$, and subject to activity-dependent adaptation or fatigue, $A$. The shape of the frequency tuning was defined by a rounded-exponential (roex) function, $W(f)=\left(1-p \frac{\left|f_{c}-f\right|}{f_{c}}\right) e^{-\frac{\left|f_{c}-f\right|}{f_{c}}}$ (Eq. 1), where $f$ is the frequency of the tone stimulus, $\left|f_{c}-f\right|$ is its absolute separation from the neuron's characteristic frequency $\left(f_{c}\right)$, and $p$ is a parameter that determines the tuning sharpness. $p$ was set such that tuning curve 
287 widths corresponded to the equivalent rectangular bandwidths (ERBs) of the auditory 288 frequency filters as determined by behavioral NN masking: $p=\frac{4 f_{V}}{\operatorname{ERE}\left(f_{V}{ }^{2}\right.}$, where $289 E R B\left(f_{c}\right)=24.7\left(4.37 f_{c}+1\right)$ (Glasberg \& Moore, 1990). The characteristic frequencies $\left(f_{c}\right)$ 290 varied from 50 to $16000 \mathrm{~Hz}$ and were distributed evenly on a cochlear frequency (ERB-rate; 291 Moore \& Glasberg, 1983) scale.

292 Adaptation was modelled by multiplying the unadapted response to the current tone 293 frequency, $f_{0}$ [given by $W\left(f_{0}\right)$; Eq. 1] with a factor $1-A$, where $A$ was proportional to the 294 response to the preceding tone frequency, $f_{-1}$ [given by $W\left(f_{-1}\right)$ ]. The degree of adaptation $(A)$ 295 was assumed to decay exponentially over time $(t): A(t)=A(t=0) e^{-t / \tau}$. The decay time 296 constant, $\tau$, was set to 721.34 ms (compare Briley \& Krumbholz, 2013; Roth et al., 1976), 297 which meant that, between successive tone onsets, adaptation decayed by $50 \%$ (because $298 e^{-500 / 721.34}=0.5$ ). The aggregate response size was derived by summing the adapted 299 single-neuron responses across neurons.

$300 \quad$ Attentional gain enhancement was modelled by multiplying the single-neuron tuning 301 functions $W$ (Eq. 1) with a gain factor, $G>1$. In the simulation shown in Fig. 1B (upper row), $302 G$ was set to 2 - doubling the attended compared to unattended response size. Attentional 303 sharpening was modelled by dividing the tuning sharpness parameter, $p$, by a sharpening 304 factor, $S<1$. In the simulations shown in Fig 1B (middle and bottom rows), $S$ was set to $0.5-$ 305 halving the ERBs of the attended compared to unattended tuning functions. If no gain is 306 applied ( $G=1$ ), halving the ERBs halves the aggregate response sizes (middle row). In order 307 to preserve the aggregate response size (bottom row), $G$ was concurrently raised to 2 .

RESULTS

\section{Behavioral results}

311 During the EEG recordings, subjects either ignored the experimental sounds and watched a 312 silent subtitled movie, or alternately monitored the tone or noise sequences in the different 313 ears for occasional target sounds (frequency-modulated tones and waning noises, 314 respectively; Fig. 1A). In order to match the difficulty in detecting the tone and noise targets, 315 each subject first attended a short pilot session, where the target salience (determined by 316 the frequency or amplitude modulation depth, respectively) was adjusted to yield a $~ 75 \%$ 317 hit rate. Across subjects, the adjusted frequency modulation depth of the tone targets 
ranged between 100-200 cents, and the amplitude modulation depth of the noise targets ranged between $50-100 \%$.

During the experiment proper, the tone targets yielded an actual hit rate close to the adjusted rate (mean \pm standard error: $76.0 \pm 3.1 \%$ ), and a false alarm rate of $10.0 \pm 2.8 \%$. In contrast, the actual hit rate for the noise targets was significantly higher $[85.0 \pm 2.8 \%$; $F(1,105)=11.3, p=0.0011 ;$ here and onwards, statistical tests are based on linear mixedeffects models (LMMs), with $\mathrm{F}$ and $\mathrm{p}$ values based on conditional F-tests; see Methods], and the false-alarm rate significantly lower $[4.4 \pm 1.3 \% ; F(1,105)=5.8 p=0.0180]$. At the same time, however, the noise targets also yielded a longer reaction time [613.6 $\pm 26.3 \mathrm{~ms}$ vs $566.0 \pm 18.0 \mathrm{~ms}$ for the tone targets; $F(1,104)=5.7 p=0.0185]$, suggesting that subjects traded response speed with response accuracy. In the case of the tone sequences, the scope for such speed-accuracy trade-off was limited by the shorter SOI (500 ms vs $816 \pm 150 \mathrm{~ms}$ for the noise sequences; see Methods), which limited the reaction time. The presence of speed-accuracy trade-off is supported by the inverse efficiency score (IES), which combines response speed and accuracy measures into a single, overall measure of task performance [IES = RT/(1-PE), where RT is the reaction time and PE is the proportion of errors, i.e., false alarms and missed targets; Townsend \& Ashby, 1978], and which was not significantly different between the tone and noise sequences [tones vs noises: $814.4 \pm 124.1 \mathrm{~ms}$ vs 829.5 $\pm 72.2 \mathrm{~ms} ; F(1,103)=0.12 p=0.7159]$. The IES was also not significantly different across the three successive 12-min measurement runs ["active runs"; main effect of run: $F(1,103)=$ $0.63, p=0.4274$; interaction between run and sequence type: $F(1,103)=0.28, p=0.5949$ ].

\section{Average CAEPs}

The average CAEPs to the non-target tones (averaged across all frequency separations between successive tones; Fig. 2A) exhibited three successive transient deflections, P1, N1 and P2, which were clearly discernible, and peaked at similar latencies (around 60, 105 and $150 \mathrm{~ms}$ ), both when the tones were attended (top panel in Fig. 2A), and when they were unattended (i.e., when subjects attended to the noise sequences in the other ear or watched a silent movie; bottom panel in Fig. 2A). Due to the relatively short SOI used (500 $\mathrm{ms})$, the CAEPs failed to return to a steady baseline before the subsequent tone onset. As a result, the transient deflections were riding on a background of slowly-varying nonstationary EEG activity from previous trials, which appeared to be particularly evident in the 
349 attended condition (Fig. 2A, top). The non-stationarity of this background activity meant 350 that it could not be eliminated by conventional baseline correction, and the use of a fixed 351 SOI (required to control the degree of adaptation between successive tones) meant that it 352 could also not be eliminated by deconvolution-based methods (Lütkenhöner, 2010; 353 Woldorff, 1993). To address this problem, we here opted to baseline-correct each deflection 354 separately, using a baseline window that was both minimal in duration and located close to 355 the respective deflection start (deflection-specific baselining; see Methods). The N1 and P2 356 were baseline-corrected to the respective preceding, opposite-polarity peak - effectively 357 creating a peak-to-peak difference. This would have minimized both the slowly-varying 358 previous-trial baseline, as well as any unipolar endogenous attention-related activity elicited within the current trial (such as the processing negativity; Näätänen, 1990). The baselinecorrected deflections are shown in Fig. 2B (separately for each attention condition) and will be referred to as $\mathrm{P} 1_{0}, N 1_{P 1}$ and $P 2_{N 1}$. Figure $3 A$ shows that they exhibited scalp voltage distributions typical of sources in supratemporal auditory cortex (characterized by a voltage inversion over the temporal bone; Scherg, Vajsar, \& Picton, 1989; Vaughan \& Ritter, 1970). $* * *$ insert Fig 2 about here $* * *$

CAEPs measured at individual sensors may reflect a mixture of contributions from both exogenous and endogenous sources, but only the exogenous contributions represent the modulatory attention effects that we aim to investigate. Thus, to maximize these contributions, we analyzed the CAEPs not only in the original sensor space (using the sensors that showed the largest unattended peak amplitude for the respective analyzed deflection; see Methods and Fig. 2B), but also in a source space representing exogenous sources. A different source model was used for each subject and analyzed deflection, based on equivalent dipoles fitted to the respective deflection peak in the individual unattended responses (where endogenous contributions should have been minimal; see Methods). Figure $3 \mathrm{~B}$ shows that the best-fitting sources for all three unattended deflections $\left(P 1_{0}, N 1_{P 1}\right.$ and $\mathrm{P} 2_{\mathrm{N} 1}$ ) localized to the approximate auditory cortex region, and that their average orientations were roughly perpendicular to the supratemporal plane. The goodness of fit ranged between 89.4 and $98.5 \%$ for the P1 [mean \pm standard deviation: $96.4 \pm 2.4$ ], between 93.7 and $98.5 \%$ for the $\mathrm{N} 1(97.1 \pm 1.4)$, and between 88.3 and $98.3 \%$ for the P2 $(95.6 \pm 2.2)$. The sources were used as spatial filters to extract source waveforms for each individual and condition (see Fig. $3 \mathrm{C}$ for the grand-average source waveforms for each 
attention condition), and the source waveforms were averaged across hemispheres, because no significant hemisphere-specific condition effects were found. ***insert Fig 3 about here***

\section{Attention effects on average CAEPs}

Comparison of the average CAEP waveforms between attention conditions (see Figs $2 B$ \& $3 C$ for the sensor and source waveforms, respectively) suggests that the $N 1_{\mathrm{P} 1}$, and, to a lesser degree, also the $\mathrm{P} 2_{\mathrm{N} 1}$, were enhanced when the tones were attended than when they were unattended, whereas the $\mathrm{P} 1_{0}$ seemed to be largely unaffected by attention. The waveforms also suggest that there was little difference between the two unattended conditions (i.e., when subjects attended to the noise sequences or watched a silent movie, labelled "ignored" and "passive" in Figs 2B \& 3C) - for any of the three deflections.

These results were confirmed by submitting the average deflection peak amplitudes (in logarithmic units; Fig. 4A) to linear mixed-effects statistical models (LMMs), with attention condition and deflection (if appropriate) as fixed factors. The models were calculated either for successive deflection pairs $\left[P 1_{0} / N 1_{P 1}\right.$ and $N 1_{P 1} / P 2_{N 1}$, or for each deflection separately (henceforth referred to as "combined" or "separate LMMs"). Effects that were significant in the current, but not the preceding deflection were interpreted as "emerging" at the level of the current deflection. Both for the sensor, and for the source data, the combined $\mathrm{LMM}$ of the $\mathrm{P} 1_{0} / \mathrm{N} 1_{\mathrm{P} 1}$ peak amplitudes revealed a significant overall (main) effect of attention condition [sensor: $F(2,107)=3.8, p=0.0254$; source: $F(2,105)=$ $6.3, p=0.0026]$, but also showed a significant deflection by attention condition interaction [sensor: $F(2,107)=15.5, p<0.0001$; source: $F(2,105)=11.1, p<0.0001]$. The interaction arose, because the attention condition effect was significant only for the $\mathrm{N}_{\mathrm{P} 1}$ [shown by the respective separate LMMs; sensor: $F(2,42)=33.0, p<0.001$; source: $F(2,42)=28.7, p<$ 0.0001 ], but non-significant for the $P 1_{0}$ [sensor: $F(2,43)=0.8, p=0.4717$; source: $F(2,41)=$ $0.4, p=0.6672]$. This suggests that the attention condition effect first emerged at the level of the $\mathrm{N} 1$. In the combined $\mathrm{LMM}$ of the $\mathrm{N} 1_{\mathrm{P} 1} / \mathrm{P} 2_{\mathrm{N} 1}$ peak amplitudes, the main effect of attention condition was again significant for both the sensor and the source data [sensor: $F(2,107)=13.3, p<0.001$; source: $F(2,105)=11.5, p<0.0001]$. In this case, the deflection by attention condition interaction was significant for the source data $[F(2,105)=5.7, p=$ $0.0045]$, but non-significant for the sensor data $[F(2,107)=0.7, p=0.5079]$. Consistent with this, the separate $\mathrm{LMM}$ for the $\mathrm{P} 2_{\mathrm{N} 1}$ showed a significant attention condition effect for the 
413 sensor data $[F(2,42)=4.1, p=0.0233]$, but not for the source data $[F(2,41)=2.12, p=$ 414 0.1274]. This suggests that the attention condition effect on the average $\mathrm{P} 2_{\mathrm{N} 1}$ peak 415 amplitudes was more labile than for the $\mathrm{N} 1_{\mathrm{P} 1}$. For the $\mathrm{N} 1_{\mathrm{P} 1}$, the attention condition effect 416 was due to larger peak amplitudes in the attended compared to both unattended (ignored 417 and passive) conditions. This was true for both the sensor (both $p \leq 0.0001$ ) and source data 418 (both $\mathrm{p}<0.0001$ ). For the $\mathrm{P} 2_{\mathrm{N} 1}$ sensor amplitudes, the difference between the attended and 419 ignored conditions was significant $(p=0.00971)$, but the difference between the attended 420 and the passive conditions was non-significant $(p=0.22429$; see stars in Fig. $4 A)$. The 421 ignored and passive conditions showed little or no differences between one another - for 422 any deflection and in either the sensor or source data (all $p>0.4$ ).

\section{Attention effects on frequency-specific adaptation}

424 To test whether the observed attention effects on the average deflection peak amplitudes 425 were generated by gain enhancement or sharpening, we evaluated the peak amplitudes 426 separately for the different frequency separations, $\Delta \mathrm{F}$, from the preceding tone, which were 427 expected to cause different degrees of adaptation (Fig. 1B). In the statistical models (LMMs) 428 frequency separation was included both as a linear $(\Delta \mathrm{F})$ and quadratic $\left(\Delta \mathrm{F}^{2}\right)$ fixed covariate, 429 because, based on the neuron population model predictions (Fig. 1B), the linear covariate 430 alone was not expected to be able to capture the effect of sharpening. For gain 431 enhancement, the model predicted a constant increase in the response size across all 432 frequency separations from the preceding tone. Statistically, this should create a main effect 433 of attention condition, with no interaction with either frequency separation covariate ( $\Delta \mathrm{F}$ or $434 \Delta \mathrm{F}^{2}$ ). In contrast, the sharpening mechanism was predicted to cause the response size 435 function to become steeper at small frequency separations, thus making the function more 436 non-linear. Statistically, this should give rise to a significant interaction between $\Delta \mathrm{F}^{2}$ and 437 attention condition. The average peak amplitudes had shown no significant differences 438 between the ignored and passive conditions for any deflection (see Fig. 4A) and the same 439 was also true for the peak amplitudes as a function of frequency separation (Fig. 4B\&C). 440 Therefore, the ignored and passive conditions were now merged to a form a single 441 "unattended" condition.

$442 * * *$ insert Fig 4 about here*** 
The $\mathrm{N} 1_{\mathrm{P} 1}$ and $\mathrm{P} 2_{\mathrm{N} 1}$ peak amplitudes increased with increasing frequency separation (Fig. $4 B \& C$ ), as predicted by the neuron population model (compare Fig. 1B). The corresponding (separate) LMMs revealed that this increase was significant for both the sensor [main effect of $\left.\Delta F ; N 1_{P 1}: F(1,241)=13.8, p=0.003 ; P 2_{N 1}: F(1,243)=15.2, p=0.0001\right]$ and source data $\left[N 1_{P 1}: F(1,241)=5.3, p=0.0221 ; P 2_{N 1}: F(1,243)=17.9, p<0.0001\right]$. These results indicate that the N1 and P2 were affected by frequency-specific adaptation. In contrast, the peak amplitudes for the $\mathrm{P} 1_{0}$ showed little or no change with frequency separation, for either the sensor [main effects of $\Delta F$ and $\Delta F^{2}$; both $F(1,235) \leq 0.5, p \geq 0.4788$ ] or source data [both $F(1,236) \leq 0.8, p \geq 0.3751]$, suggesting that the $P 1$ was either not adapted, or that adaptation in the $\mathrm{P} 1$ was non-specific to frequency.

Figure $4 \mathrm{~B} \& \mathrm{C}$ (middle panels) suggests that attention increased the $\mathrm{N} 1_{\mathrm{P} 1}$ peak amplitudes about equally across all frequency separations. This finding was statistically confirmed by the non-significance of the interactions between attention condition and both $\Delta \mathrm{F}$ and $\Delta \mathrm{F}^{2}$ in the separate $L M M$ for the $N 1_{p 1}$, which applied to both the sensor [both $F(1,241) \leq 1.3, p \geq$ 0.2558 ] and source data [both $\mathrm{F}(1,241) \leq 2.5, \mathrm{p} \geq 0.1164$ ], and is consistent with the neuron population model predictions for gain enhancement (compare upper right panel in Fig. 1B). In contrast, the attention effect on the $\mathrm{P} 2_{\mathrm{N} 1}$ peak amplitudes depended strongly on frequency separation, with little or no increase at the zero and largest frequency separations ( 0 and 300 cents; 100 cents correspond to 1 semitone), but large increases at the intervening frequency separations (75 and 150 cents; rightmost panels in Fig. 4B\&C). This pattern is consistent with the neuron population model predictions for sharpening combined with a commensurate gain enhancement to preserve the aggregate response size (compare bottom right panel in Fig. 1B). Statistically, it was confirmed by the significance of the interaction between attention condition and $\Delta \mathrm{F}^{2}$ in the separate $\mathrm{LMM}$ for the $\mathrm{P} 2_{\mathrm{N} 1}$, which, again, applied to both the sensor $[F(1,243)=11.0, p=0.001]$ and source data $[F(1,243)=5.0, p=0.0264]$. The interaction between attention condition and $\Delta F$ was nonsignificant [sensor: $F(1,243)=0.4, p=0.5292$; source: $F(1,243)=0.2, p=0.6822$ ]

The difference in the pattern of frequency separation-dependent attention effects between the $\mathrm{N} 1_{\mathrm{P} 1}$ and $\mathrm{P} 2_{\mathrm{N} 1}$ was statistically confirmed by the three-way interaction 472 between deflection, attention condition and $\Delta \mathrm{F}^{2}$ in the corresponding combined LMM $473\left(\mathrm{~N} 1_{\mathrm{P} 1} / \mathrm{P} 2_{\mathrm{N} 1}\right)$. This interaction, which was significant in the sensor data $[\mathrm{F}(1,506)=8.2, \mathrm{p}=$ 
$4740.0045]$ and approached significance in the source data $[F(1,506)=3.2, p=0.0764]$, 475 suggesting that sharpening emerges only at the level of the $\mathrm{P} 2$. In contrast to the $\mathrm{N} 1_{\mathrm{P} 1}$ and $476 \mathrm{P} 2_{\mathrm{N} 1}$, the $\mathrm{P} 1_{0}$ peak amplitudes showed no significant attention effects, at any frequency 477 separation, as confirmed by the lack of significant interactions between attention condition 478 and both $\Delta \mathrm{F}$ or $\Delta \mathrm{F}^{2}$ in the separate LLMs for the $\mathrm{P} 1_{0}$ [sensor: both $\mathrm{F}(1,235) \leq 0.1, \mathrm{p} \geq 0.7699$; 479 source: both $F(1,236) \leq 1.4, p \geq 0.2427]$.

\section{DISCUSSION}

482 The current results suggest that the earliest effects of auditory attentional modulation 483 are mediated by a pure gain enhancement mechanism, and that sharpening emerges only at 484 later processing stages. In the current results, the earliest measured deflection, the P1 485 presumed to be generated in primary auditory cortex (Liégeois-Chauvel, Musolino, Badier, 486 Marquis, \& Chauvel, 1994; Mäkelä, Hämäläinen, Hari, \& McEvoy, 1994; Yvert, Crouzeix, 487 Bertrand, Seither-Preisler, \& Pantev, 2001) - was little or not affected by attention. The 488 subsequent N1 showed a strong attention-related enhancement in average peak amplitude, 489 but no differential effects on frequency-specific adaptation, suggesting that the N1 was 490 affected by a pure gain enhancement mechanism. In contrast, the latest measured 491 deflection, the P2, showed a lesser enhancement in average peak amplitude, but a marked 492 increase in the degree of adaptation specificity. Predictions from a neuron population model showed that the pattern of the effects in the P2 was consistent with a sharpening in neural tuning selectivity, combined with a commensurate gain enhancement so that the overall response size remained unchanged.

These results are consistent with previous studies that have also found large attentional enhancements in N1 peak amplitude (Hillyard et al., 1973; Neelon, Williams, \& Garell, 2006a, 2006b), but contradict the conclusion of the previous NN masking studies (Ahveninen et al., 2011; Kauramaki et al., 2007; Okamoto et al., 2007) that attentional enhancement of the N1 is caused by neuronal sharpening. In the NN studies, attention was directed to a specific frequency value and the audibility of the attended stimulus was allowed to vary across conditions. As explained above (Introduction) this would likely have led to variation in the amount of attentional gain enhancement, in a way that would have mimicked the expected effect of sharpening (Alho et al., 1992; Boudreau et al., 2006; Schwent et al., 1976a, 1976b). In the current study, attention was directed to one or other 
ear, and stimulus audibility was fixed across conditions. Our results thus suggest that attention can sharpen selectivity for a feature (here, frequency) even when attention is not selectively focused on a specific feature value. A similar conclusion was reached by Murray and Wojciulik (2004), who used an adaptation paradigm to demonstrate attentional sharpening for visual orientation. In both our and Murray and Wojciulik's studies, the feature in which sharpening was observed (frequency and visual orientation, respectively) was task-relevant (in Murray and Wojciulik's study, subjects had to detect a change in image orientation; in our study, they had to detect a small frequency modulation). It is thus possible that task relevance is a prerequisite for sharpening to occur.

$$
\text { The absence of significant attention effects in the earliest, P1, deflection in the current }
$$
study is consistent with several previous studies (Hillyard et al., 1973; Neelon, Williams, \& Garell, 2006a, 2006b) that have also found no significant P1 attention effects. Other studies, however, that have used shorter SOls, did find significant attention effects in the P1, and even earlier, deflections (Woldorff et al., 1993; Woldorff \& Hillyard, 1991), suggesting that the first emergence of attention effects is graded with attentional load.

The current finding of a small but significant (in the sensor data) attentional enhancement in the average P2 peak amplitude contrasts with some previous CAEP studies that have found either no significant change (Hillyard et al., 1973) or even a reduction (Hansen \& Hillyard, 1980) in the P2 amplitude as a result of attention. The reduction has been attributed to a separate unipolar deflection, termed the "processing negativity" or "Nd", thought to reflect endogenous attention-related processes (Näätänen, 1990). Due to its negative polarity, the $\mathrm{Nd}$ would be expected to add to any modulatory enhancement of the N1, but diminish any enhancement of the P2. In the current study, this effect would have been minimized by the deflection-specific baselining procedure used (see Methods). Significant attentional enhancement of the $\mathrm{P} 2$ has also been found in intracranial recordings from the auditory temporal region (Neelon et al., 2006a, 2006b), where any influence of the $\mathrm{Nd}$ may also have been minimal. The $\mathrm{Nd}$ can be demonstrated by calculating the difference wave between attended and unattended responses. In the current study, this was precluded by the experimental design: Difference waves can only be meaningfully calculated when the previous-trial baseline activity in the attended and unattended responses is either the same on average (e.g., Hansen \& Hillyard, 1983; Hillyard \& Münte, 1984), or can be effectively corrected for (e.g., Woldorff \& Hillyard, 1991; Woldorff, 1993). In the current study, 
538 attended and unattended trials were temporally separated into different blocks, and so, 539 attended trials were always preceded by attended trials, and unattended trials were always 540 preceded by unattended trials. As a result, the attended responses exhibited a substantially 541 larger previous-trial baseline, on average, than the unattended responses. Correcting for the 542 baseline was also not possible, as this requires a sufficiently variable SOI (Lütkenhöner, 543 2010; Woldorff, 1993). In the current study, the SOI had to be fixed to control the degree of 544 adaptation between successive trials.

545 The N1 and P2 have often been viewed as part of the same component process (the so546 called "N1-P2 complex"). However, the marked differences in the pattern of their observed 547 attention effects suggests that, rather than representing a unitary complex, the N1 and P2 548 represent different hierarchical levels of exogenous auditory processing that play distinct 549 functional roles in conscious sound perception. This is supported by previous findings 550 showing that the N1 and P2 differ not only in source structure (Godey, Schwartz, de Graaf, 551 Chauvel, \& Liégeois-Chauvel, 2001; Hari, Kaila, Katila, Tuomisto, \& Varpula, 1982; Hari et al., 552 1987; Lütkenhöner \& Steinsträter, 1998), but also in functional properties, such as 553 dependence on prior stimulation, general arousal, aging and auditory training (Crowley \& 554 Colrain, 2004; Herrmann, Henry, Johnsrude, \& Obleser, 2016; Ross, Jamali, \& Tremblay, 555 2013; Ross \& Tremblay, 2009; Roth et al., 1976; Tremblay, Ross, Inoue, McClannahan, \& Collet, 2014).

The effect of attention on adaptation, or "repetition suppression", has been investigated by several previous studies - particularly in the visual domain and using fMRI (see Henson \& Mouchlianitis, 2007, for review). The results from these studies, however, have been mixed, with some studies finding similar repetition suppression in both attended and unattended conditions (Bentley, Vuilleumier, Thiel, Driver, \& Dolan, 2003; Vuilleumier, Schwartz, 562 Duhoux, Dolan, \& Driver, 2005), but others finding repetition suppression to be either 563 reduced (Murray \& Wojciulik, 2004) or absent in unattended conditions (Eger, Henson, 564 Driver, \& Dolan, 2004; Henson \& Mouchlianitis, 2007; Yi, Kelley, Marois, \& Chun, 2006; Yi, 565 Woodman, Widders, Marois, \& Chun, 2004). The previous studies compared responses to 566 repeated versus different stimuli, but, unlike the current study, did not vary the degree of 567 stimulus difference. The current results suggest that the amount of unattended repetition 568 suppression should depend on the relation between the degree of stimulus difference and 569 neuronal tuning selectivity: if we had compared repeated versus different tones with only a 
single frequency separation, we would have observed similar attended and unattended repetition suppression, if the frequency separation had been greater than 150 cents, but reduced or absent unattended repetition suppression if the frequency separation had been equal to or smaller than 150 cents (see Fig. $4 B \& C$ ).

Previous studies from the visual (Summerfield, Trittschuh, Monti, Mesulam, \& Egner, 2008; Summerfield, Wyart, Johnen, \& de Gardelle, 2011) and auditory (Todorovic, van Ede, Maris, \& de Lange, 2011; Wacongne et al., 2011) domains have demonstrated that repetition suppression is not only determined by the local stimulus context (locally preceding stimuli), but is also modulated by prior expectation, such that the amount of repetition suppression is reduced when stimulus repetition is unexpected. This is contrary to the idea of bottom-up neuronal fatigue, and has been taken to suggest that repetition suppression may instead reflect the action of a hierarchical predictive coding mechanism, which combines bottom-up stimulus representations with prior, top-down stimulus expectations (e.g., Friston, 2005; Knill \& Pouget, 2004). Within this predictive coding framework, it has been hypothesized that attention may modulate the top-down stimulus expectations - increasing expectation for attended over unattended stimuli (Friston, 2009; Rao, 2005). Several recent studies have interpreted their findings within the context of this hypothesis (Chennu et al., 2013; Hsu, Hämäläinen, \& Waszak, 2014; Kok, Rahnev, Jehee, Lau, \& de Lange, 2012). The current study, however, suggests an alternative, or at least complementary, explanation. This is, because all stimuli and all stimulus transitions (including higher-order transitions between non-consecutive stimuli) were perfectly balanced (see Methods), and thus presumably equally expected - and attention was also distributed equally across all stimuli. This excludes an explanation in terms of top-down expectation, and instead suggests that attention modulates bottom-up representational properties.

The P2 amplitude showed little or no attention-related change when the frequency separation from the preceding tone was either zero or larger. According to the neuron population model predictions, this suggest that the P2 was affected by a combination of sharpening and gain enhancement, and that the amount of gain enhancement matched the degree of sharpening, such that the overall response size remained unchanged. This suggests that gain enhancement and sharpening are distinct but cooperative components of a hierarchically distributed attentional modulation mechanism, which adaptively adjusts the 
representational bandwidth of auditory cortical processing in accordance with attentional

demand. Sharpening increases representational resolution, but, without a commensurate enhancement in gain, this would lead to decrease in representational accuracy (because fewer channels would be activated, or each channel would be activated less strongly). By combining and matching gain enhancement and sharpening effects, the auditory system can increase representational resolution whilst, at the same time, maintaining representational accuracy. And by cascading the gain enhancement and sharpening effects across different processing levels - presumably with different limitations on representational resources (Ahissar \& Hochstein, 2004), the system retains the ability to quickly switch attention to new, or currently unattended, sounds.

\section{ACKNOWLEDGEMENTS}

This work was funded by the MRC (intramural programme grants MC_U135097128 and MC_UU00010/2). We thank Sarah Jane Gibbs for her help in participant screening and data collection, and Oliver Zobay for assistance with the statistical analysis.

\section{REFERENCES}

617

618

619

620

621

622

623

624

625

626

627

628

629

630

631

632

633

634

635

636

637

638
Ahissar, M., \& Hochstein, S. (2004). The reverse hierarchy theory of visual perceptual learning. Trends in Cognitive Sciences, 8(10), 457-464.

Ahveninen, J., Hämäläinen, M., Jääskeläinen, I. P., Ahlfors, S. P., Huang, S., Lin, F. H., et al. (2011). Attention-driven auditory cortex short-term plasticity helps segregate relevant sounds from noise. Proceedings of the National Academy of Sciences of the United States of America, 108(10), 4182-4187.

Alho, K., Woods, D. L., Algazi, A., \& Näätänen, R. (1992). Intermodal selective attention. II. Effects of attentional load on processing of auditory and visual stimuli in central space. Electroencephalography and Clinical Neurophysiology, 82(5), 356-368.

Atiani, S., Elhilali, M., David, S. V., Fritz, J. B., \& Shamma, S. A. (2009). Task difficulty and performance induce diverse adaptive patterns in gain and shape of primary auditory cortical receptive fields. Neuron, 61(3), 467-480.

Bentley, P., Vuilleumier, P., Thiel, C. M., Driver, J., \& Dolan, R. J. (2003). Effects of attention and emotion on repetition priming and their modulation by cholinergic enhancement. Journal of Neurophysiology, 90(2), 1171-1181.

Boudreau, C. E., Williford, T. H., \& Maunsell, J. H. (2006). Effects of task difficulty and target likelihood in area V4 of macaque monkeys. Journal of Neurophysiology, 96(5), 2377-2387.

Briley, P. M., Breakey, C., \& Krumbholz, K. (2013). Evidence for pitch chroma mapping in human auditory cortex. Cerebral Cortex, 23(11), 2601-2610.

Briley, P. M., \& Krumbholz, K. (2013). The specificity of stimulus-specific adaptation in human auditory cortex increases with repeated exposure to the adapting stimulus. Journal of Neurophysiology, 110(12), 2679-2688. 
Brimijoin, W. O., \& O'Neill, W. E. (2010). Patterned tone sequences reveal non-linear interactions in auditory spectrotemporal receptive fields in the inferior colliculus. Hearing Research, 267(12), 96-110.

Chennu, S., Noreika, V., Gueorguiev, D., Blenkmann, A., Kochen, S., Ibáñez, A., et al. (2013). Expectation and attention in hierarchical auditory prediction. The Journal of Neuroscience, 33(27), 11194-11205.

Crowley, K. E., \& Colrain, I. M. (2004). A review of the evidence for P2 being an independent component process: age, sleep and modality. Clinical Neurophysiology, 115(4), 732-744.

Cusack, R., Deeks, J., Aikman, G., \& Carlyon, R. P. (2004). Effects of location, frequency region, and time course of selective attention on auditory scene analysis. Journal of Experimental Psychology. Human Perception and Performance, 30(4), 643-656.

Edmonds, B. A., \& Krumbholz, K. (2014). Are interaural time and level differences represented by independent or integrated codes in the human auditory cortex? Journal of the Association for Research in Otolaryngology, 15(1), 103-114.

Eger, E., Henson, R. N., Driver, J., \& Dolan, R. J. (2004). BOLD repetition decreases in objectresponsive ventral visual areas depend on spatial attention. Journal of Neurophysiology, 92(2), 1241-1247.

Fox, J., \& Weisberg, S. (2011). An R Companion to Applied Regression (Second ed.). Los Angeles, CA: Sage.

Friston, K. (2005). A theory of cortical responses. Philosophical Transactions of the Royal Society of London. Series B, Biological Sciences, 360(1456), 815-836.

Friston, K. (2009). The free-energy principle: a rough guide to the brain? Trends in Cognitive Sciences, 13(7), 293-301.

Fritz, J. B., Elhilali, M., David, S. V., \& Shamma, S. A. (2007). Auditory attention--focusing the searchlight on sound. Current Opinion in Neurobiology, 17(4), 437-455.

Fritz, J. B., Shamma, S., Elhilali, M., \& Klein, D. (2003). Rapid task-related plasticity of spectrotemporal receptive fields in primary auditory cortex. Nature Neuroscience, 6(11), 1216-1223.

Fujiwara, N., Nagamine, T., Imai, M., Tanaka, T., \& Shibasaki, H. (1998). Role of the primary auditory cortex in auditory selective attention studied by whole-head neuromagnetometer. Brain Research. Cognitive Brain Research, 7(2), 99-109.

Glasberg, B. R., \& Moore, B. C. J. (1990). Derivation of auditory filter shapes from notched-noise data. Hearing Research, 47(1-2), 103-138.

Glasberg, B. R., \& Moore, B. C. J. (2000). Frequency selectivity as a function of level and frequency measured with uniformly exciting notched noise. The Journal of the Acoustical Society of America, 108(5 Pt 1), 2318-2328.

Godey, B., Schwartz, D., de Graaf, J. B., Chauvel, P., \& Liégeois-Chauvel, C. (2001). Neuromagnetic source localization of auditory evoked fields and intracerebral evoked potentials: a comparison of data in the same patients. Clinical Neurophysiology, 112(10), 1850-1859.

Grill-Spector, K., Henson, R., \& Martin, A. (2006). Repetition and the brain: neural models of stimulus-specific effects. Trends in Cognitive Sciences, 10(1), 14-23.

Hansen, J. C., \& Hillyard, S. A. (1980). Endogenous brain potentials associated with selective auditory attention. Electroencephalography and Clinical Neurophysiology, 49(3-4), 277-290.

Hansen, J. C., \& Hillyard, S. A. (1983). Selective attention to multidimensional auditory stimuli. Journal of Experimental Psychology: Human Perception and Performance, 9(1), 1-19.

Hari, R., Kaila, K., Katila, T., Tuomisto, T., \& Varpula, T. (1982). Interstimulus interval dependence of the auditory vertex response and its magnetic counterpart: implications for their neural generation. Electroencephalography and Clinical Neurophysiology, 54(5), 561-569.

Hari, R., Pelizzone, M., Mäkelä, J. P., Hällström, J., Leinonen, L., \& Lounasmaa, O. V. (1987). Neuromagnetic responses of the human auditory cortex to on- and offsets of noise bursts. Audiology, 26(1), 31-43. 
Henson, R. N., \& Mouchlianitis, E. (2007). Effect of spatial attention on stimulus-specific

Herrmann, B., Henry, M. J., Johnsrude, I. S., \& Obleser, J. (2016). Altered temporal dynamics of neural adaptation in the aging human auditory cortex. Neurobiology of Aging, 45, 10-22.

Hewson-Stoate, N., Schonwiesner, M., \& Krumbholz, K. (2006). Vowel processing evokes a large sustained response anterior to primary auditory cortex. The European Journal of Neuroscience, 24(9), 2661-2671.

Hillyard, S. A., \& Münte, T. F. (1984). Selective attention to color and location: An analysis with event-related brain potentials. Attention, Perception, \& Psychophysics, 36(2), 185-198.

Hillyard, S. A., Hink, R. F., Schwent, V. L., \& Picton, T. W. (1973). Electrical signs of selective attention in the human brain. Science, 182(4108), 177-180.

Hillyard, S. A., Vogel, E. K., \& Luck, S. J. (1998). Sensory gain control (amplification) as a mechanism of selective attention: electrophysiological and neuroimaging evidence. Philosophical Transactions of the Royal Society of London. Series B, Biological Sciences, 353(1373), 12571270.

Hothorn, T., Bretz, F., \& Westfall, P. (2008). Simultaneous inference in general parametric models. Biometrical Journal, 50(3), 346-363.

Hsu, Y. F., Hämäläinen, J. A., \& Waszak, F. (2014). Repetition suppression comprises both attentionindependent and attention-dependent processes. Neurolmage, 98, 168-175.

Jäncke, L., Mirzazade, S., \& Shah, N. J. (1999). Attention modulates activity in the primary and the secondary auditory cortex: a functional magnetic resonance imaging study in human subjects. Neuroscience Letters, 266(2), 125-128.

Kauramaki, J., Jääskeläinen, I. P., \& Sams, M. (2007). Selective attention increases both gain and feature selectivity of the human auditory cortex. PloS One, 2(9), e909.

Knill, D. C., \& Pouget, A. (2004). The Bayesian brain: the role of uncertainty in neural coding and computation. Trends in Neurosciences, 27(12), 712-719.

Kok, P., Rahnev, D., Jehee, J. F., Lau, H. C., \& de Lange, F. P. (2012). Attention reverses the effect of prediction in silencing sensory signals. Cerebral Cortex, 22(9), 2197-2206.

Lanting, C. P., Briley, P. M., Sumner, C. J., \& Krumbholz, K. (2013). Mechanisms of adaptation in human auditory cortex. Journal of Neurophysiology, 110(4), 973-983.

Liégeois-Chauvel, C., Musolino, A., Badier, J. M., Marquis, P., \& Chauvel, P. (1994). Evoked potentials recorded from the auditory cortex in man: evaluation and topography of the middle latency components. Electroencephalography and Clinical Neurophysiology, 92(3), 204-214.

Lütkenhöner, B. (2010). Baseline correction of overlapping event-related responses using a linear deconvolution technique. Neurolmage, 52(1), 86-96.

Lütkenhöner, B., \& Steinsträter, O. (1998). High-precision neuromagnetic study of the functional organization of the human auditory cortex. Audiology \& Neuro-Otology, 3(2-3), 191-213.

Magezi, D. A., \& Krumbholz, K. (2010). Evidence for opponent-channel coding of interaural time differences in human auditory cortex. Journal of Neurophysiology, 104(4), 1997-2007.

Mäkelä, J. P., Hämäläinen, M., Hari, R., \& McEvoy, L. (1994). Whole-head mapping of middle-latency auditory evoked magnetic fields. Electroencephalography and Clinical Neurophysiology, 92(5), 414-421.

Mangun, G. R., \& Hillyard, S. A. (1995). Mechanisms and models of selective attention. In M. D. Rugg \& M. G. H. Coles (Eds.), Electrophysiology of Mind: Event-related Brain Potentials and Cognition (pp. 40-85). New York, NY: Oxford University Press.

McAdams, C. J., \& Maunsell, J. H. (1999). Effects of attention on orientation-tuning functions of single neurons in macaque cortical area V4. The Journal of Neuroscience, 19(1), 431-441.

Moore, B. C. J., \& Glasberg, B. R. (1983). Suggested formulae for calculating auditory-filter bandwidths and excitation patterns. The Journal of the Acoustical Society of America, 74(3), 750-753. 
Murray, S. O., \& Wojciulik, E. (2004). Attention increases neural selectivity in the human lateral occipital complex. Nature Neuroscience, 7(1), 70-74.

Näätänen, R. (1990). The Role of Attention in Auditory Information-Processing as Revealed by EventRelated Potentials and Other Brain Measures of Cognitive Function. Behavioral and Brain Sciences, 13(2), 201-232.

Näätänen, R., \& Picton, T. (1987). The N1 wave of the human electric and magnetic response to sound: a review and an analysis of the component structure. Psychophysiology, 24(4), 375425.

Neelon, M. F., Williams, J., \& Garell, P. C. (2006a). The effects of attentional load on auditory ERPs recorded from human cortex. Brain Research, 1118(1), 94-105.

Neelon, M. F., Williams, J., \& Garell, P. C. (2006b). The effects of auditory attention measured from human electrocorticograms. Clinical Neurophysiology, 117(3), 504-521.

Okamoto, H., Stracke, H., Wolters, C. H., Schmael, F., \& Pantev, C. (2007). Attention improves population-level frequency tuning in human auditory cortex. The Journal of Neuroscience, 27(39), 10383-10390.

Pinheiro, J., \& Bates, D. (2000). Mixed-Effects Models in S and S-PLUS. New York, NY: Springer

Pinheiro, J., Bates, D., DebRoy, S., Sarkar, D., \& Team, R. C. (2017). nlme: Linear and Nonlinear Mixed Effects Models. from https://CRAN.R-project.org/package $=$ nlme

R Core Team. (2013). A Language and Environment for Statistical Computing. from http://www.Rproject.org

Rao, R. P. (2005). Bayesian inference and attentional modulation in the visual cortex. Neuroreport, 16(16), 1843-1848.

Ross, B., Jamali, S., \& Tremblay, K. L. (2013). Plasticity in neuromagnetic cortical responses suggests enhanced auditory object representation. BMC Neuroscience, 14, 151.

Ross, B., \& Tremblay, K. (2009). Stimulus experience modifies auditory neuromagnetic responses in young and older listeners. Hearing Research, 248(1-2), 48-59.

Roth, W. T., Krainz, P. L., Ford, J. M., Tinklenberg, J. R., Rothbart, R. M., \& Kopell, B. S. (1976). Parameters of temporal recovery of the human auditory evoked potential. Electroencephalography and Clinical Neurophysiology, 40(6), 623-632.

Salminen, N. H., May, P. J., Alku, P., \& Tiitinen, H. (2009). A population rate code of auditory space in the human cortex. PloS One, 4(10), e7600.

Sams, M., \& Salmelin, R. (1994). Evidence of sharp frequency tuning in the human auditory cortex. Hearing Research, 75(1-2), 67-74.

Scherg, M., \& Ebersole, J. S. (1993). Models of brain sources. Brain Topography, 5(4), 419-423.

Scherg, M., Vajsar, J., \& Picton, T. W. (1989). A source analysis of the late human auditory evoked potentials. Journal of Cognitive Neuroscience, 1(4), 336-355.

Schwent, V. L., Hillyard, S. A., \& Galambos, R. (1976a). Selective attention and the auditory vertex potential. Effects of signal intensity and masking noise. Electroencephalography and Clinical Neurophysiology, 40(6), 615-622.

Schwent, V. L., Hillyard, S. A., \& Galambos, R. (1976b). Selective attention and the auditory vertex potential. I. Effects of stimulus delivery rate. Electroencephalography and Clinical Neurophysiology, 40(6), 604-614.

Snow, M., Coen-Cagli, R., \& Schwartz, O. (2017). Adaptation in the visual cortex: a case for probing neuronal populations with natural stimuli F1000Research, 6 .

Spitzer, H., Desimone, R., \& Moran, J. (1988). Increased attention enhances both behavioral and neuronal performance. Science, 240(4850), 338-340.

Summerfield, C., Trittschuh, E. H., Monti, J. M., Mesulam, M. M., \& Egner, T. (2008). Neural repetition suppression reflects fulfilled perceptual expectations. Nature Neuroscience, 11(9), 1004-1006. 
Summerfield, C., Wyart, V., Johnen, V. M., \& de Gardelle, V. (2011). Human Scalp Electroencephalography Reveals that Repetition Suppression Varies with Expectation. Frontiers in Human Neuroscience, 5, 67.

Todorovic, A., van Ede, F., Maris, E., \& de Lange, F. P. (2011). Prior expectation mediates neural adaptation to repeated sounds in the auditory cortex: an MEG study. The Journal of Neuroscience, 31(25), 9118-9123.

Townsend, J. T., \& Ashby, F. G. (1978). Methods of modeling capacity in simple processing systems. In J. Castellan \& F. Restle (Eds.), Cognitive Theory (Vol. 3, pp. 199-239). Hillsdate, NJ: Erlbaum Associates.

Tremblay, K. L., Ross, B., Inoue, K., McClannahan, K., \& Collet, G. (2014). Is the auditory evoked P2 response a biomarker of learning? Frontiers in Systems Neuroscience, 8, 28.

Vaughan, H. G., Jr., \& Ritter, W. (1970). The sources of auditory evoked responses recorded from the human scalp. Electroencephalography and Clinical Neurophysiology, 28(4), 360-367.

Vuilleumier, P., Schwartz, S., Duhoux, S., Dolan, R. J., \& Driver, J. (2005). Selective attention modulates neural substrates of repetition priming and "implicit" visual memory: suppressions and enhancements revealed by FMRI. Journal of Cognitive Neuroscience, 17(8), 1245-1260.

Wacongne, C., Labyt, E., van Wassenhove, V., Bekinschtein, T., Naccache, L., \& Dehaene, S. (2011). Evidence for a hierarchy of predictions and prediction errors in human cortex. Proceedings of the National Academy of Sciences of the United States of America, 108(51), 20754-20759.

Webster, M. A. (2015). Visual Adaptation. Annual Review of Vision Science, 1, 547-567.

Woldorff, M. G. (1993). Distortion of ERP averages due to overlap from temporally adjacent ERPs: analysis and correction. Psychophysiology, 30(1), 98-119.

Woldorff, M. G., Gallen, C. C., Hampson, S. A., Hillyard, S. A., Pantev, C., Sobel, D., et al. (1993). Modulation of early sensory processing in human auditory cortex during auditory selective attention. Proceedings of the National Academy of Sciences of the United States of America, 90(18), 8722-8726.

Woldorff, M. G., \& Hillyard, S. A. (1991). Modulation of early auditory processing during selective listening to rapidly presented tones. Electroencephalography and Clinical Neurophysiology, 79(3), 170-191.

Yi, D. J., Kelley, T. A., Marois, R., \& Chun, M. M. (2006). Attentional modulation of repetition attenuation is anatomically dissociable for scenes and faces. Brain Research, 1080(1), 53-62.

Yi, D. J., Woodman, G. F., Widders, D., Marois, R., \& Chun, M. M. (2004). Neural fate of ignored stimuli: dissociable effects of perceptual and working memory load. Nature Neuroscience, 7(9), 992-996.

Yvert, B., Crouzeix, A., Bertrand, O., Seither-Preisler, A., \& Pantev, C. (2001). Multiple supratemporal sources of magnetic and electric auditory evoked middle latency components in humans. Cerebral Cortex, 11(5), 411-423. 


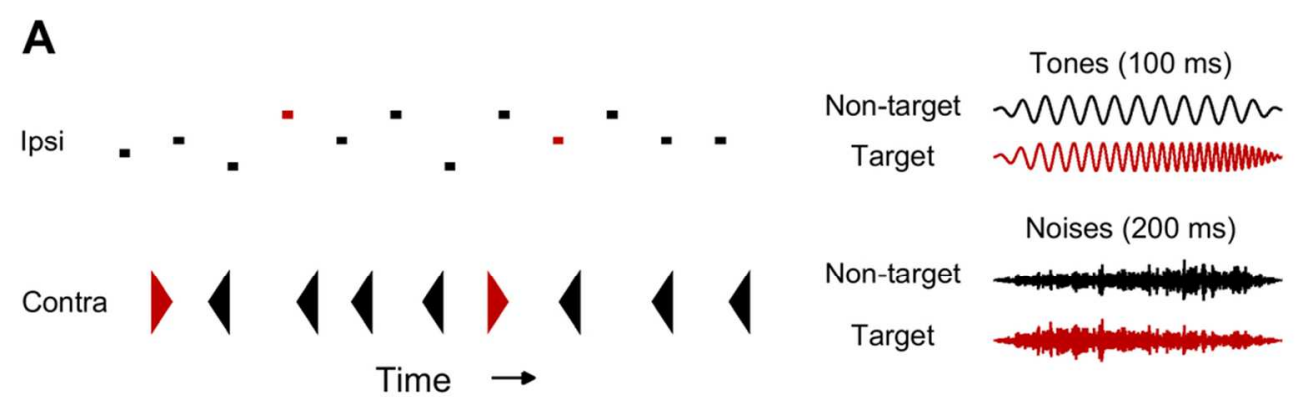

\section{B}

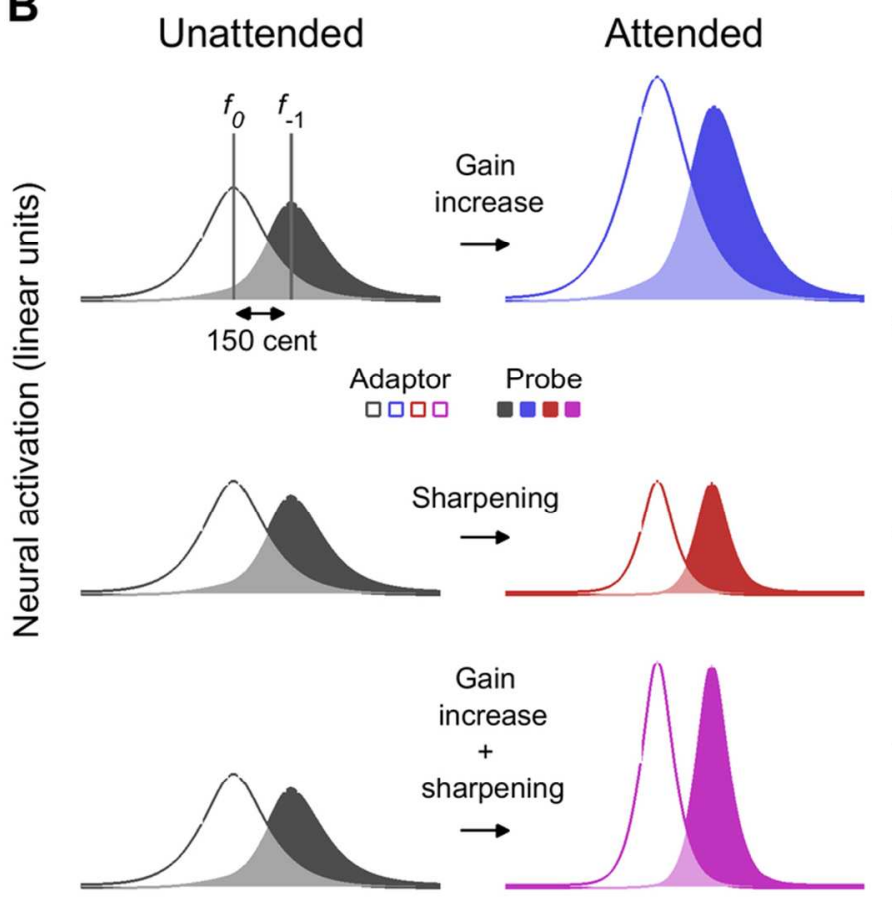

Characteristic frequency (quasi-log units)

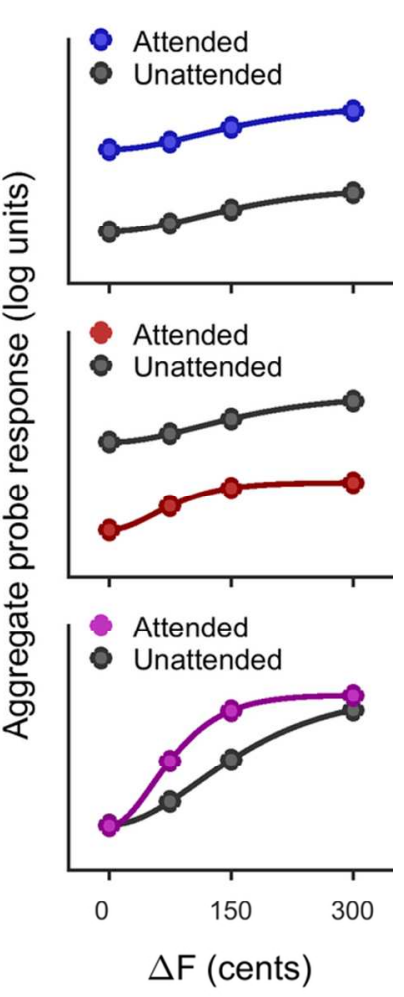

Figure 1: Experimental design and neuron population model predictions. (A) Dichotic attention task: one ear received rapid sequences of sine tones varying pseudo-randomly between four different frequencies ("Ipsi"), and the other received sequences of waxing noises ("Contra"). Subjects were instructed to attend to either sequence alternately ( $\sim 2$ min cycle) and detect rare target sounds (shown in red; non-targets are shown in black) in the attended sequence. The tone targets were distinguished by an upward-sweeping frequency trajectory, and the noise targets by a time-reversed (waning) amplitude envelope (see sound examples on the right; tone frequencies and stimulus durations not to scale). (B) Neuron population model predictions of attentional gain enhancement and sharpening (see Methods). The top and middle rows show the predicted effects of gain enhancement and sharpening separately, and the bottom row shows the effect of sharpening combined with a gain increase to counteract suppression in the aggregate response size. The left and middle columns show simulated population activity distributions to the adapting and adapted tones (open and filled outlines), plotted as a function of neuron characteristic frequency in quasi-logarithmic (cochlear frequency) units. The right column shows the aggregate sizes of the adapted responses as a function of the adaptorprobe frequency separation. The unattended responses are shown in gray (replotted across rows), and the attended responses in color. 
A

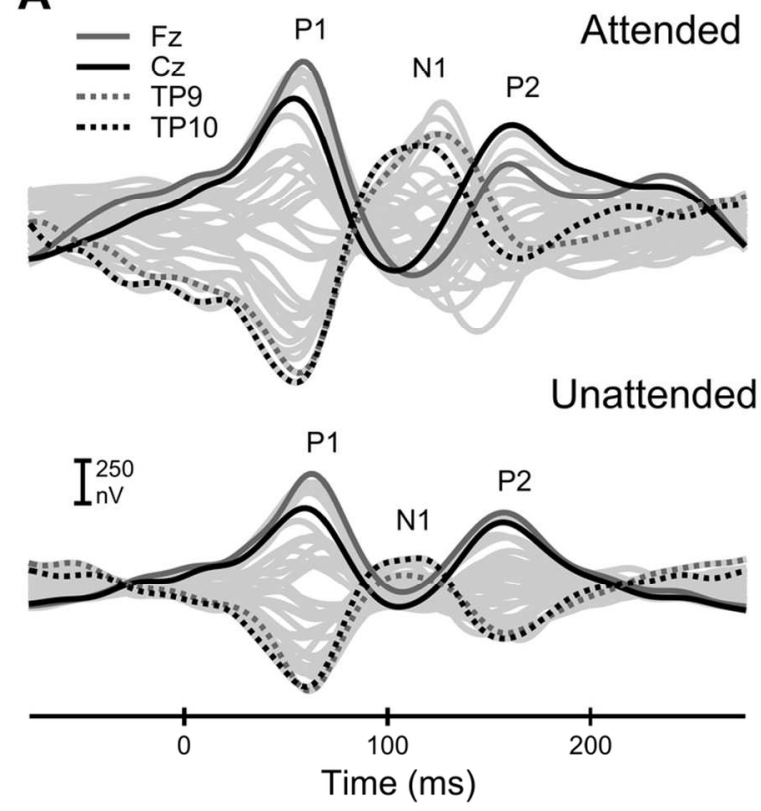

B
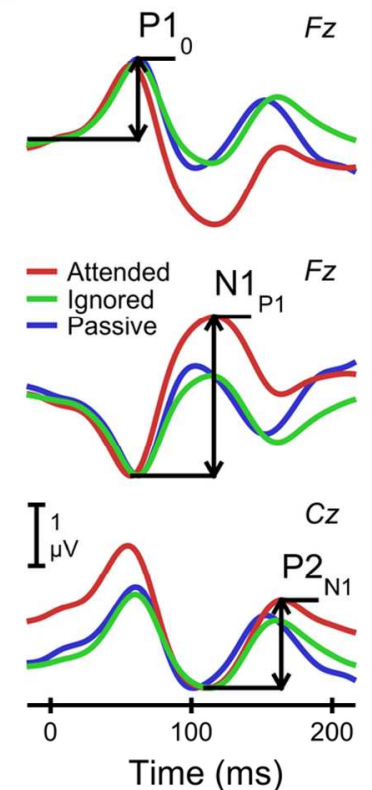

Figure 2: Cortical auditory-evoked potentials (CAEPs). (A) Grand-average CAEPs across subjects and tone frequencies in the attended (top) and unattended (bottom) conditions, shown as a butterfly plot of all 33 sensors (gray lines). The colored lines highlight the sensors with the largest positive (fronto-central and central; Fz and Cz) and negative (left and right mastoids; TP9 and TP10) deflection amplitudes. No baseline correction was applied to these responses. (B) Baseline-corrected grand-average CAEPs for the P1 (P10; top), N1 (N1P1; middle) and P2 (P2N1; bottom) and each attention condition (attended, ignored and passive; see legend). The waveforms shows the sensors with the largest positive amplitude for each deflection (Fz for P10 and N1P1, Cz for the P2N1), referenced to the average of the mastoid sensors (TP9 and TP10). The vertical arrows show the baseline-corrected peak amplitudes for the attended condition. 
A
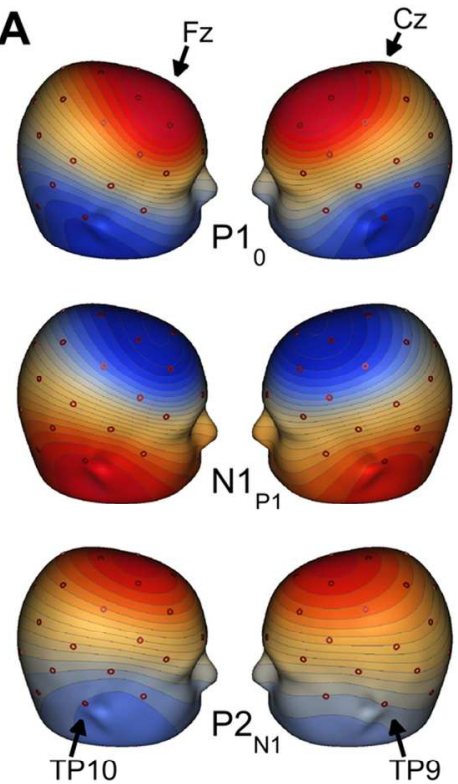

B
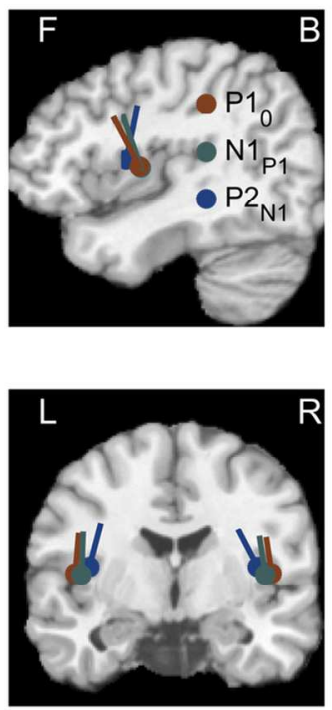

C
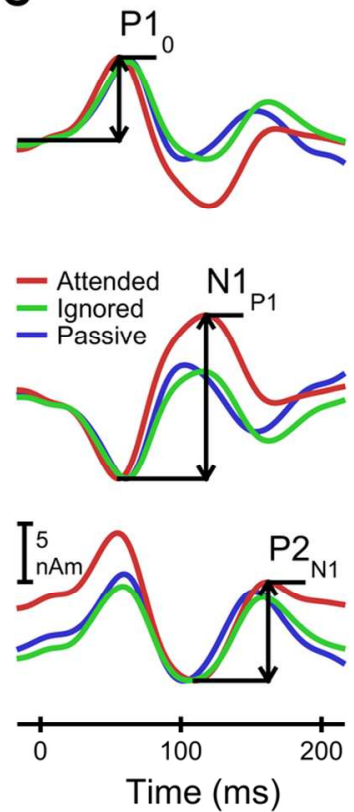

Figure 3: CAEP source analysis. (A) Scalp voltage distributions at the peak latencies of the baselinecorrected P10, N1P1 and P2N1 deflections, averaged over all unattended trials (ignored and passive conditions). (B) Source locations of symmetric equivalent current dipole (ECD) pairs, fitted to each deflection peak (see Methods). The dipole locations (dots) and orientations (short lines) are shown on sagittal (top) and coronal (bottom) slices of the single-subject MNI template brain. (C) Grand-average source waveforms for the P10 (top), N1P1 (middle) and P2N1 (bottom) deflections in each attention condition. The vertical arrows show the baseline-corrected peak amplitudes for the attended condition. 

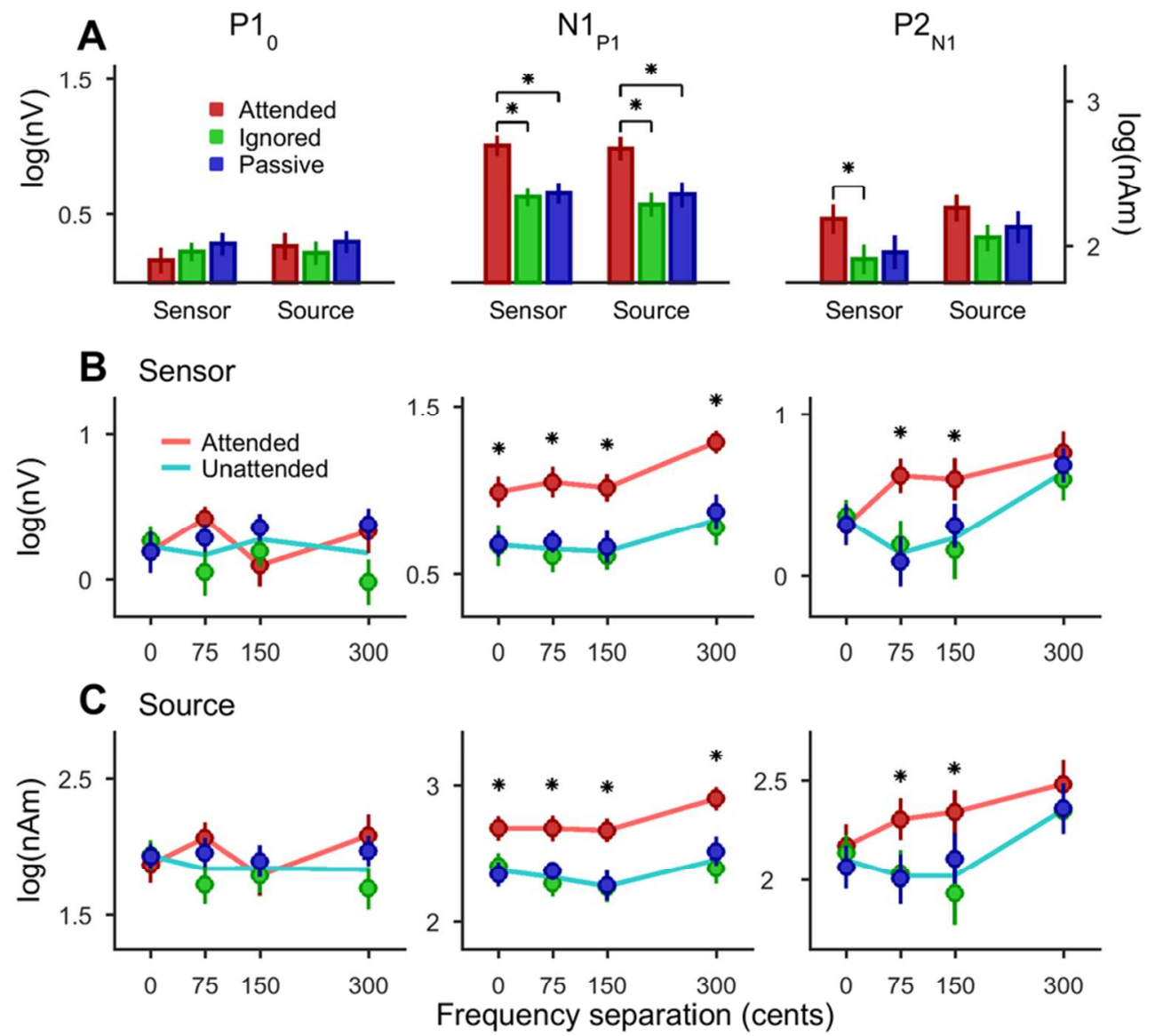

Figure 4: Attention effects on CAEP peak amplitudes. (A) Attention effect of the average deflection peak amplitudes. Different attention conditions are indicated by different colors (see legend), and different deflections are shown in different panels. In each panel, the left set of bars shows the results for the sensor data, and the right set of bars shows the results for the source data. (B) Attention effect on the sensorbased deflection peak amplitudes as a function of the frequency separation from the preceding tone (expressed as absolute value in cents, where 100 cents $=1$ semitone). The different attention conditions are shown by different colors as in A. (C) Same as in (B), but for the source-based deflection peak amplitudes. In all panels, the mean deflection peak amplitudes are expressed in logarithmic units, and the error bars show the logarithmic standard error of the mean. 